\title{
Proliferative Glomerulonephritis
}

National Cancer Institute

\section{Source}

National Cancer Institute. Proliferative Glomerulonephritis. NCI Thesaurus. Code C35281.

A constellation of renal disorders characterized by an increase number of cells in the glomerulus; these disorders generally present with nephrotic syndrome, and generally progress to end stage renal failure over a matter of weeks to years, depending on the etiology. Examples include IgA nephropathy, membranoproliferative glomerulonephritis, and rapidly progressive glomerulonephritis. 